\title{
The etiology of uterine sarcomas: a pooled analysis of the epidemiology of endometrial cancer consortium
}

A S Felix ${ }^{\star}, 1,2$, L S Cook ${ }^{3}$, M M Gaudet ${ }^{4}$, T E Rohan ${ }^{5}$, L J Schouten ${ }^{6}$, V W Setiawan ${ }^{7}$, L A Wise ${ }^{8}$, K E Anderson ${ }^{9}$, L Bernstein ${ }^{10}$, I De Vivo ${ }^{11,12}$, C M Friedenreich ${ }^{13}$, S M Gapstur ${ }^{4}$, R A Goldbohm ${ }^{14}$, B Henderson ${ }^{7}, \mathrm{P}$ L Horn-Ross ${ }^{15}$, L Kolone ${ }^{16}$, J V Lacey ${ }^{10}$, X Liang ${ }^{17}$, J Lissowska ${ }^{18}$, A Magliocco $^{19}$, M L McCullough ${ }^{4}$, A B Miller ${ }^{20}$, S H Olson ${ }^{17}$, J R Palmer ${ }^{8}$, Y Park $^{2}$, A V Patel ${ }^{4}$, J Prescott ${ }^{11,12}$, R Rastogi ${ }^{17}$, K Robien ${ }^{9}$, L Rosenberg ${ }^{8}$, C Schairer ${ }^{2}$, X Ou Shu ${ }^{21}$, P A van den Brandt ${ }^{6}$, R A Virkus ${ }^{8}$, N Wentzensen ${ }^{2}$, Y-B Xiang ${ }^{22}, \mathrm{~W}-\mathrm{H} X \mathrm{u}^{22}, \mathrm{H} \mathrm{P}$ Yang $^{2}$ and L A Brinton ${ }^{2}$

${ }^{1}$ Cancer Prevention Fellowship Program, Division of Cancer Prevention, National Cancer Institute, National Institutes of Health, Rockville, MD, USA; ${ }^{2}$ Division of Cancer Epidemiology and Genetics, National Cancer Institute, National Institutes of Health, Rockville, MD, USA; ${ }^{3}$ Division of Epidemiology and Biostatistics, Department of Internal Medicine, University of New Mexico, Albuquerque, NM, USA; ${ }^{4}$ Epidemiology Research Program, American Cancer Society, Atlanta, GA, USA; ${ }^{5}$ Epidemiology and Population Health, Albert Einstein College of Medicine, Bronx, NY, USA; ${ }^{6}$ GROW-School for Oncology and Developmental Biology, Maastricht University, Maastricht, The Netherlands; ${ }^{7}$ Department of Preventive Medicine, University of Southern California, Los Angeles, CA, USA; ${ }^{8}$ Slone Epidemiology Center at Boston University, Boston, MA, USA; ${ }^{9}$ Division of Epidemiology and Community Health, University of Minnesota, Minneapolis, MN, USA; ${ }^{10}$ Department of Population Sciences, Beckman Research Institute, City of Hope, Duarte, CA, USA; ${ }^{11}$ Channing Division of Network Medicine, Department of Medicine, Brigham and Women's Hospital and Harvard Medical School, Boston, MA, USA; ${ }^{2}$ Program in Molecular and Genetic Epidemiology, Department of Epidemiology, Harvard School of Public Health, Boston, MA, USA; ${ }^{13}$ Division of Cancer Care, Alberta Health Services-Cancer Care, Edmonton, Alberta, Canada; ${ }^{14}$ Department of Prevention and Health, Netherlands Organisation for Applied Scientific Research (TNO), Leiden, The Netherlands; ${ }^{15}$ Cancer Prevention Institute of California, Fremont, CA, USA; ${ }^{16}$ Epidemiology Program, University of Hawaii Cancer Center, Honolulu, HI, USA; ${ }^{17}$ Epidemiology and Biostatistics, Memorial Sloan-Kettering Cancer Center, New York, NY, USA; ${ }^{18}$ M Sklodowska-Curie Memorial Cancer Center and Institute of Oncology, Warsaw, Poland; ${ }^{19}$ Department of Anatomical Pathology, H. Lee Moffitt Cancer Center, Tampa, FL, USA; ${ }^{20}$ Dalla Lana School of Public Health, University of Toronto, Toronto, Ontario, Canada; ${ }^{21}$ Department of Medicine, Vanderbilt University Medical Center, Nashville, TN, USA and ${ }^{22}$ Department of Epidemiology, Shanghai Cancer Institute, Shanghai, China

Background: Uterine sarcomas are characterised by early age at diagnosis, poor prognosis, and higher incidence among Black compared with White women, but their aetiology is poorly understood. Therefore, we performed a pooled analysis of data collected in the Epidemiology of Endometrial Cancer Consortium. We also examined risk factor associations for malignant mixed mullerian tumours (MMMTs) and endometrioid endometrial carcinomas (EECs) for comparison purposes.

Methods: We pooled data on 229 uterine sarcomas, 244 MMMTs, 7623 EEC cases, and 28829 controls. Odds ratios (ORs) and 95\% confidence intervals $(\mathrm{Cls})$ for risk factors associated with uterine sarcoma, MMMT, and EEC were estimated with polytomous logistic regression. We also examined associations between epidemiological factors and histological subtypes of uterine sarcoma.

Results: Significant risk factors for uterine sarcoma included obesity (body mass index (BMI) $\geqslant 30$ vs BMl<25 kg m ${ }^{-2}(\mathrm{OR}: 1.73,95 \% \mathrm{Cl}: 1.22-2.46$ ), $P$-trend $=0.008)$ and history of diabetes (OR: $2.33,95 \% \mathrm{Cl}: 1.41-3.83)$. Older age at menarche was inversely associated with uterine sarcoma risk ( $\geqslant 15$ years vs $<11$ years (OR: $0.70,95 \% \mathrm{Cl}: 0.34-1.44$ ), P-trend: 0.04$)$. BMI was significantly, but less strongly related to uterine sarcomas compared with EECs (OR: 3.03, 95\% Cl: 2.82-3.26) or MMMTs (OR: 2.25, 95\% Cl: 1.60-3.15, P-heterogeneity $=0.01$ ).

Conclusion: In the largest aetiological study of uterine sarcomas, associations between menstrual, hormonal, and anthropometric risk factors and uterine sarcoma were similar to those identified for EEC. Further exploration of factors that might explain patterns of age- and race-specific incidence rates for uterine sarcoma are needed.

*Correspondence: Dr AS Felix; E-mail: ashley.felix@nih.gov

Received 8 October 2012; revised 13 December 2012; accepted 16 December 2012; published online 24 January 2013

(c) 2013 Cancer Research UK. All rights reserved 0007-0920/13 
Uterine sarcoma is a rare form of uterine cancer that arises from the myometrium or connective tissue of the uterus and accounts for $3-7 \%$ of all uterine cancer diagnoses in the United States (D'Angelo and Prat, 2010). Unlike the most common uterine cancer histological type, endometrioid endometrial carcinoma (EEC), uterine sarcomas are highly aggressive, with 5-year overall survival rates ranging between 17 and 55\% (Prat, 2009). The peak incidence of uterine sarcoma occurs at a younger age than EEC and several studies reported higher incidence rates of uterine sarcoma among Black compared with White women (Harlow et al, 1986; Schwartz et al, 1996; Brooks et al, 2004), the opposite of overall endometrial carcinoma trends (Sherman and Devesa, 2003).

Owing to the low incidence of this disease, the aetiology of uterine sarcomas has been investigated in only a few small casecontrol studies (Kvale et al, 1988; Schwartz and Thomas, 1989; Schwartz and Weiss, 1990; Schwartz et al, 1991, 1996; Lavie et al, 2008; Jaakkola et al, 2011). Obesity, menopausal use of oestrogen plus progestin, oral contraceptives (OC), and tamoxifen use are associated with increased risks of uterine sarcoma, whereas cigarette smoking and parity are associated with a reduced risk. Recently, there was an important change in the classification of uterine sarcoma; malignant mixed mullerian tumours (MMMTs), which previously accounted for $40 \%$ of all uterine sarcomas, are now classified as metaplastic endometrial carcinomas given their similarities in aetiology and metastatic patterns (McCluggage, 2002; Prat, 2009). Consequently, previous risk factor associations may have been affected by the inclusion of the MMMT subtype. Here, we examine relationships between epidemiological risk factors and uterine sarcoma, overall and by histological subtype, in a large pooled analysis using the updated histological classification for uterine sarcoma. Furthermore, we examine risk factor associations for MMMTs and EECs to evaluate potential aetiologic heterogeneity across a spectrum of uterine cancer diagnoses.

\section{MATERIALS AND METHODS}

Study population. The Epidemiology of Endometrial Cancer Consortium (E2C2), sponsored in part by the National Cancer Institute, was designed to combine data from cohort and casecontrol studies to elucidate the aetiology of uterine cancer (Olson et al, 2009). Any study that included at least one uterine sarcoma case was eligible for the current analysis. The 10 cohort and five case-control studies that contributed data to this analysis are summarised in Table 1. For the cohort studies that contributed data to E2C2 (other than the California Teachers Study (CTS)), a nested case-control study design was employed, with inclusion of up to four controls (women with an intact uterus and no uterine cancer diagnosis) randomly selected from the risk set and matched to the corresponding uterine cancer case on year of birth, date of entry (within 6 months), and any additional matching criteria as appropriate in the individual study. For the CTS, data came from a previous nested case-control study in which two controls per case were identified and matching was based on 5-year age group, race/ ethnicity, and broad geographic area within California. Cases in the cohort studies were identified through annual linkage to state or national cancer registries (Multiethnic Cohort Study, NIH-AARP Diet and Health Study (NIH-AARP), Iowa Women's Health Study (IWHS), Netherlands Cohort Study (NLCS), Canadian National Breast Screening Study (NBSS), and CTS) or by self-report on follow-up questionnaires and confirmed through medical record review, linkage to cancer registries, or the National Death Index (Cancer Prevention Study II Nutrition Cohort, Breast Cancer Detection Demonstration Project (BCDDP), Nurses' Health Study (NHS), and Black Women's Health Study (BWHS)).

In the case-control studies, population-based controls were frequency-matched to cases except in the US Case-Control Study
(US) where individual 1:1 matching was employed. Eligible controls were those women with an intact uterus and no history of uterine cancer. Methods to select controls within each source population included random digit dialling (US, Bay Area Womens Health Study (BAWHS), Endometrial Cancer and Physical Activity Study (ECPA)) and random selection from data registrars of all citizens (Polish Endometrial Cancer Study (PECS) and Shanghai Endometrial Cancer Study (SECS)). All studies were approved by the institutional review boards (IRBs) of their parent institutions, and written informed consent was obtained from all participants. In addition, Memorial Sloan-Kettering Cancer Centre has IRB approval as the data coordinating centre for $\mathrm{E} 2 \mathrm{C} 2$.

Data collection. De-identified data from the participating studies were centrally collected and harmonised at Memorial SloanKettering Cancer Centre. We made an effort to collect a core set of standardized variables, but not all variables were collected by each study. Some studies did not provide information on menopausal oestrogen plus progestin use (NBSS, ECPA, NLCS, IWHS, BCDDP), menopausal oestrogen-alone use (NBSS, ECPA, IWHS), diabetes (BAWHS and NBSS), parity (NLCS), or smoking status (BAWHS). As the number of live births was not reported by the NLCS, we used the number of pregnancies lasting $\geqslant 7$ months as a surrogate for parity among NLCS cases and controls.

Case definitions. Women with an incident, histologically confirmed diagnosis of uterine sarcoma, MMMT, or EEC were included as case patients in the current study. Although the emphasis of this study is on uterine sarcomas, women with MMMTs or EECs were included for comparison purposes. Uterine sarcoma cases with the following International Classification of Diseases for Oncology (ICD)-O-3 morphology codes were included: sarcoma, not otherwise specified (NOS) 8800-8806, fibromatous neoplasms 8810-8815, myomatous neoplasms 8890 8896 (includes leiomyosarcoma), rhabdomyosarcoma 8900-8902, embryonal rhabdomyosarcoma 8910-8912, and endometrial stromal sarcoma 8930-8934. Four studies (PECS, SECS, BWHS, and NHS) did not have ICD-O-3 codes and instead supplied a summary histology variable for each case (i.e., sarcoma, EEC, MMMT, etc). The ICD-O-3 codes $8950-8982$ or summary variable 'MMMT' were used to define MMMT, while ICD-O-3 codes 8380-8383 and summary variable 'endometrioid' identified EECs. EEC cases from the NHS could not be distinguished from adenocarcinoma, NOS cases and were excluded from analysis.

Statistical methods. Categories for exposure variables were created including age ( $\leqslant 54,55-59,60-64,65-69, \geqslant 70$ years), race (White, Black, Asian, other), BMI ( $<25,25-30$, $\left.\geqslant 30 \mathrm{~kg} \mathrm{~m}^{-2}\right)$, age at menarche $(<11,11-12,13-14, \geqslant 15$ years $)$, menopausal status (premenopausal, peri-menopausal, postmenopausal), parity (no live births, 1 or more live births), number of live births among parous women $(1,2,3-4, \geqslant 5$ live births), smoking status (never, former, current), menopausal hormone use (never, ever), menopausal oestrogen use (never, ever), menopausal oestrogen plus progestin use (never, ever), OC use (never, ever), and history of diabetes (no, yes). Given the importance of these variables in the aetiology of common endometrial carcinoma subtypes, we included all exposure variables simultaneously in an unconditional polytomous logistic regression model to estimate the magnitude of association (odds ratios (ORs) and 95\% confidence intervals (CIs)) between risk factors and case groups. Polytomous logistic regression was used when the outcome variable is nominal with more than two levels (Hosmer, 2000). When a study did not report values for a particular variable, that study was excluded from the specific risk factor analysis. Missing values were coded as a separate category for each variable; when excluding subjects with missing values the results did not appreciably change. 


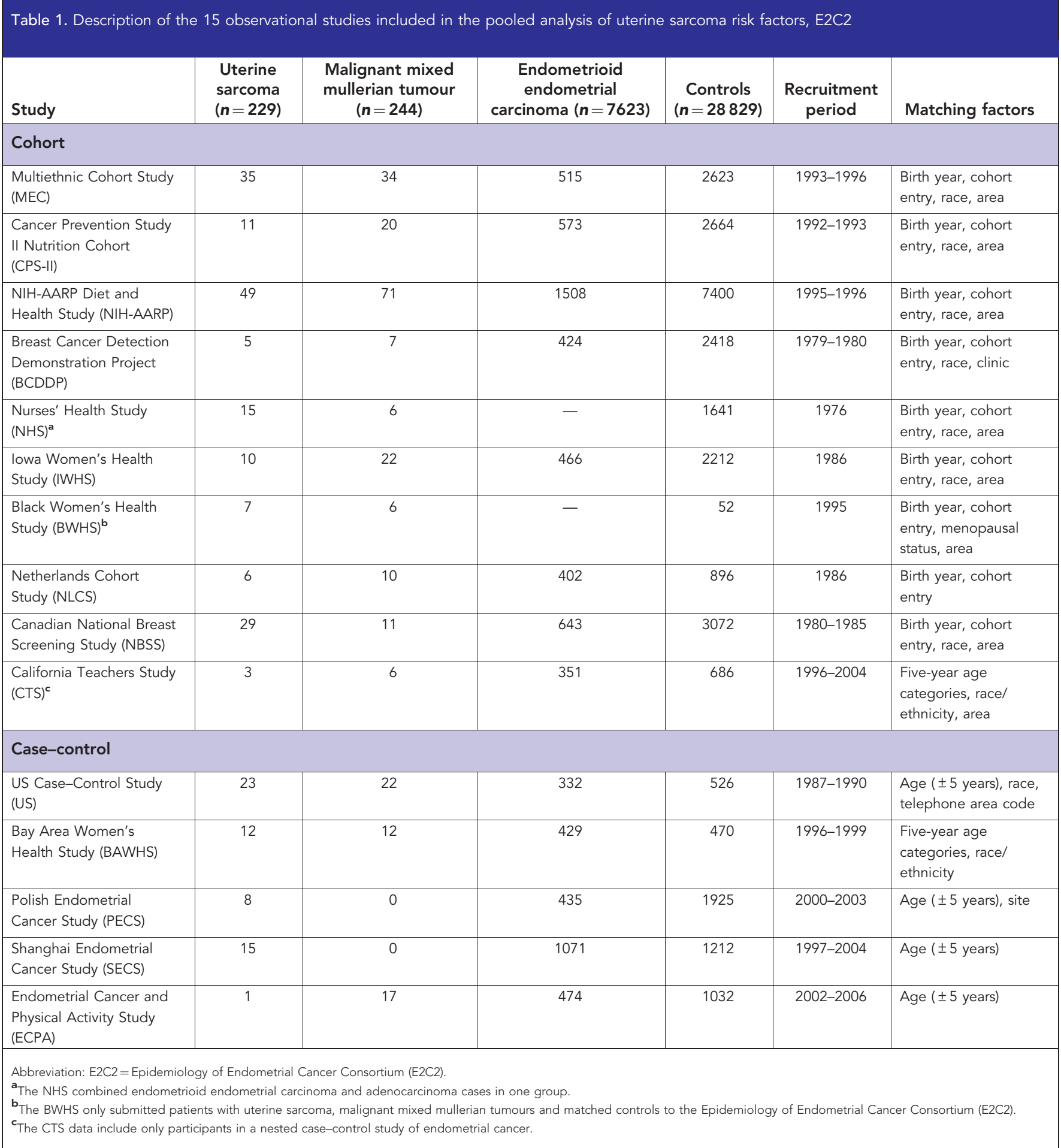

All models were adjusted for age and race; however, we do not present effect estimates for these variables given their use as matching criteria in all studies. Tests for linear trend were performed for BMI, age at menarche, and number of live births among parous women by including the ordinal form of each variable in the model. We also examined risk factors for endometrial stromal sarcoma and leiomyosarcoma, the two main histological subtypes of uterine sarcoma, compared with controls. Differences in ORs between case groups were quantified using case-only logistic regression models. A $P$-heterogeneity $<0.05$ indicated the magnitude of effect for a particular risk factor was significantly different between case groups. Between-study heterogeneity of effect estimates was examined by creating a multiplicative interaction term between study site (fixed effect covariate) and each risk factor and performing a likelihood ratio test comparing models with and without the risk factor-study site interaction terms.

Using the distribution of risk factors in our sample, a binary outcome (control $v s$ uterine sarcoma), power of $80 \%$ and a two-sided $\alpha$ of 0.05 , we calculated minimum detectable ORs for each risk factor, which ranged from 1.45-1.89 for factors associated with increased risk and $0.35-0.67$ for protective factors. All tests of statistical significance were two-sided. Analyses were performed using SAS version 9.3 (SAS Institute Inc., Cary, NC, USA). 


\section{RESULTS}

A total of 229 uterine sarcomas, 244 MMMTs, 7623 EECs, and 28829 controls were available for this pooled analysis. Black race was more prevalent among MMMTs compared with uterine sarcoma and EEC cases $(17.2 \%, 11.3 \%$, and $2.6 \%$, respectively, data not tabled), and median age at diagnosis was oldest among MMMT cases compared with uterine sarcoma and EEC cases (67.0, 61.4, and 64.3 years, respectively, data not tabled). Distributions of risk factors, ORs, and 95\% CIs are shown in Table 2. Significantly increased risk of uterine sarcoma was observed for obese compared to normal BMI (OR: 1.73, 95\% CI: $1.22-2.46)$ and a history of diabetes compared with no diabetes (OR: 2.33, 95\% CI: 1.41-3.83), whereas older age at menarche (age at menarche $\geqslant 15$ compared with age at menarche $<11$ years OR: $0.70,95 \%$ CI: $0.34-1.44$, p-trend $=0.04$ ) was associated with a lower risk of uterine sarcoma. Any live births, postmenopausal status, OC use, and current or former smoking were inversely but not statistically significantly associated with uterine sarcoma risk. BMI was significantly, but less strongly related to uterine sarcoma than to EECs (OR: 3.03, 95\% CI: 2.82-3.26) or MMMTs (OR: 2.25, 95\% CI: 1.60-3.15) for the heaviest compared with the leanest women $(P$-heterogeneity $=0.01)$.

In exploratory analyses, we examined risks associated with the most prevalent histological subtypes of uterine sarcoma: endometrial stromal sarcoma $(n=98)$ and leiomyosarcoma $(n=82)$ (Table 3). Black race was more prevalent among leiomyosarcoma compared with endometrial stromal sarcoma cases $(20.7 \%$ vs $6.1 \%$, data not tabled), whereas median age at diagnosis was similar $(61.8$ and 63.6 years, respectively, data not tabled). The direction of most associations for the histological subtypes was similar to patterns observed for uterine sarcoma overall. Obesity (OR: 1.74, 95\% CI: 1.03-2.93) and a history of diabetes (OR: 2.28, 95\% CI: $1.02-5.12$ ) were associated with significantly higher risks of endometrial stromal sarcoma, whereas reduced risk of leiomyosarcoma was observed for postmenopausal compared with premenopausal women (OR: 0.35 , 95\% CI: 0.16-0.75). Compared with the overall associations, less consistency in histological subtype associations was noted for age at menarche and former or current smoking. However, no significant heterogeneity of effects between these two histological subtypes was observed $(P$-heterogeneity $>0.10)$.

\section{DISCUSSION}

The present pooled analysis examined the association between previously identified endometrial carcinoma risk factorsincluding reproductive, hormonal, and anthropometric factorsand uterine sarcoma, a rare yet fatal uterine cancer subtype. Our data suggest that uterine sarcoma shares certain risk factors with EECs but less so with MMMTs. Similar to EEC, obesity and history of diabetes were linked with an increased risk of uterine sarcoma, while older age at menarche was associated with decreased risk. Subtype analyses of endometrial stromal sarcoma and leiomyosarcoma generally revealed risk factor associations similar to those observed for all uterine sarcomas combined.

Uterine sarcomas fall under the broad category of soft tissue sarcomas, which are extremely rare regardless of the site of origin. Previously documented risk factors for this heterogeneous group of tumours include ionising radiation, exposure to certain chemicals, and genetic syndromes, such as neurofibromatosis type 1 and Li-Fraumeni syndrome (Skubitz and D'Adamo, 2007). Uterine sarcomas have been particularly difficult to examine owing to changes in classification over time, histological diversity, and low incidence rates. In 2009, the International Federation of Gynaecology and Obstetrics reclassified MMMTs, which at the time was the most common uterine sarcoma histology subtype (40\%), as a metaplastic endometrial carcinoma. Moreover, the remaining uterine sarcoma subtypes-leiomyosarcoma, endometrial stromal sarcoma, adenosarcoma, and undifferentiated sarcoma-are a heterogeneous group, which complicates the study of their aetiology. The two most common subtypes now, leiomyosarcoma and endometrial stromal sarcoma, can be distinguished by their sarcomatous appearance during histology examination. However, expert pathologists are needed for the correct classification of these subtypes (Chu et al, 2001).

Our results support findings from some previous studies on risk factors for specific histological subtypes of uterine sarcoma. In a case-control study (167 cases, 208 controls), Schwartz et al (1991, 1996) reported on uterine sarcoma subtype risks associated with exogenous hormone use, obesity, smoking, and menstrual and reproductive characteristics. As in our study, high BMI was associated with increased risks of endometrial stromal sarcoma $(n=26)$, while the risk of leiomyosarcoma $(n=56)$ was lower among cigarette smokers than never smokers. Although we observed inverse associations with current smoking status for uterine sarcoma overall (OR: 0.88) and for leiomyosarcoma (OR: $0.75)$, these associations did not achieve statistical significance. Prior reports also suggest decreased uterine sarcoma risk associated with older age at menarche (Kvale et al, 1988; Schwartz et al, 1991), which was apparent in our study for uterine sarcoma risk overall and of the endometrial stromal sarcoma subtype. Parity was associated with decreased uterine sarcoma risk in a prior study (Kvale et al, 1988); however, our results concur with two other reports that did not observe clear associations with any live births, the number of live births, and uterine sarcoma risk (Schwartz and Thomas, 1989; Schwartz et al, 1991).

In contrast to prior reports, we observed statistically nonsignificant inverse associations between OC use and uterine sarcoma risk overall, as well as risk of both histological subtypes, whereas Schwartz et al. (1996) reported positive, albeit, statistically nonsignificant associations. Given the absence of statistical significance and information on the formulation and duration of OC use in ours and the previous study, these findings should be interpreted cautiously. Furthermore, we did not observe an association between menopausal oestrogen plus progestin use and uterine sarcoma risk, which has been observed previously. In a recent Finnish cohort study, menopausal estradiol and progestin treatment was associated with increased risks of leiomyosarcoma and endometrial stromal sarcoma, especially among women with longer exposures (Jaakkola et al, 2011). Finally, the relationship between a history of diabetes and uterine sarcoma risk has been explored in one previous study (Brinton et al, 2005). Of 137 uterine sarcoma cases, only 2 had a history of diabetes resulting in a null association. We noted strong risks associated with a history of diabetes for uterine sarcoma overall and both histological subtypes, which is consistent with aetiologic studies of endometrial carcinoma (Weiderpass et al, 2000; Rosato et al, 2011). Obesity and diabetes are associated with metabolic disturbances and our finding of a stronger association with diabetes for uterine sarcoma compared with EEC raises questions about the possibility of a more central role of insulin in their aetiology.

Similarities in risk factor associations for uterine sarcoma and EECs suggest overlap in the biological mechanisms associated with development of these tumours. Commonly described mechanisms relating menstrual, reproductive, and anthropometric factors to EEC risk include imbalances in multiple pathways, including sex hormones (oestrogen and progesterone), insulin and insulin-like growth factors (IGFs), and inflammatory markers such as interleukins. Higher expression of oestrogen, IGFs, and interleukins is associated with increased risk of EECs (Calle and Kaaks, 2004; Oh et al, 2004; Dossus et al, 2010; Audet-Walsh et al, 2011; Wang et al, 2011). Key cytogenetic and molecular events observed in endometrial stromal sarcomas include chromosomal rearrangements, loss of heterozygosity of tumour suppressor genes, and 


\begin{tabular}{|c|c|c|c|c|c|c|c|c|c|c|c|c|c|c|c|}
\hline & 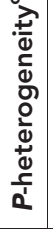 & os. & & 然 & & ¿. & & $\stackrel{\circ}{0}$ & & 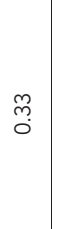 & & ठิ & & 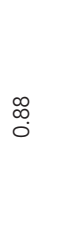 & \\
\hline 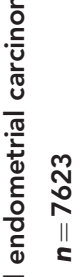 & 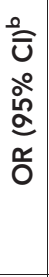 & & 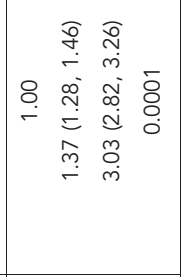 & & 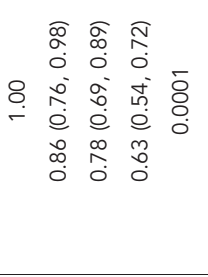 & & 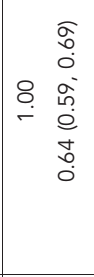 & & 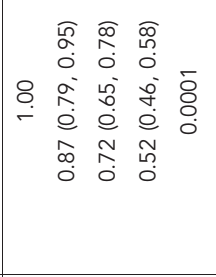 & & 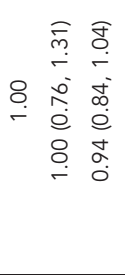 & & 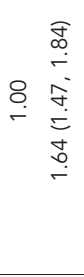 & & 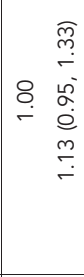 \\
\hline & $\circ$ & & 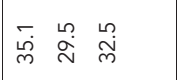 & & 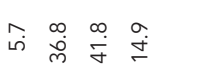 & & $\begin{array}{ll}0 & \hat{0} \\
\stackrel{\infty}{0}\end{array}$ & & 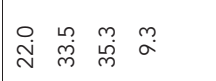 & & 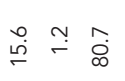 & & 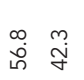 & & 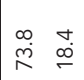 \\
\hline & $=$ & & 号 & & 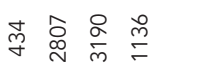 & & $\stackrel{\circ}{\stackrel{2}{\sim}}$ & & 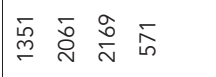 & & $\stackrel{\infty}{\infty} \approx \underset{\sim}{\sim}$ & & 命 & & $\underset{m}{9} \bar{\alpha}$ \\
\hline 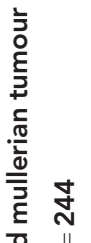 & 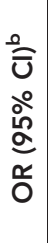 & & 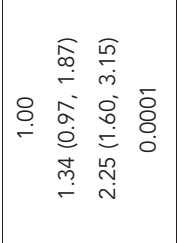 & & 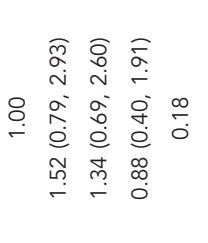 & & 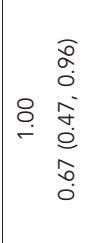 & & 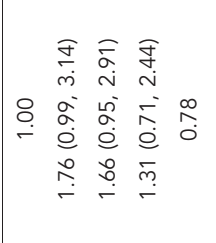 & & 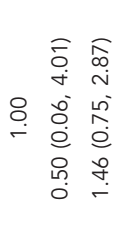 & & 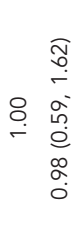 & & 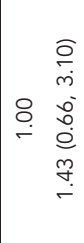 \\
\hline 离 & $\circ$ & & $\hat{i}$ 官管 & & 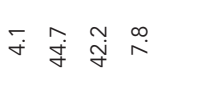 & & 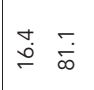 & & 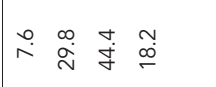 & & ஸि & & 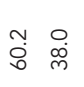 & & $\stackrel{\circ}{\stackrel{\circ}{ } \stackrel{?}{ }}$ \\
\hline & $=$ & & $\stackrel{\circ}{\circ} \stackrel{+}{\infty}$ & & 웅 용 & & $g \stackrel{\infty}{\circ}$ & & $\stackrel{n}{\llcorner} \stackrel{\infty}{\infty} \infty \stackrel{\infty}{\infty}$ & & $\pm-\bar{N}$ & & $\stackrel{m}{m} \underset{\infty}{\infty}$ & & $\stackrel{\tilde{m}}{\bar{m}}$ \\
\hline 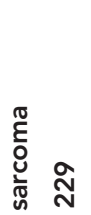 & 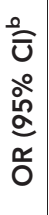 & & 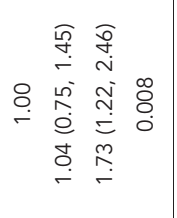 & & 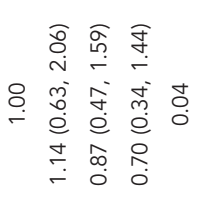 & & 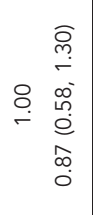 & & 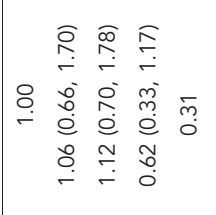 & & 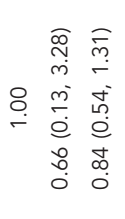 & & 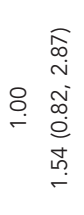 & & 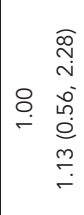 \\
\hline 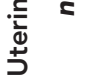 & ๑ீ & & 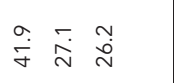 & & 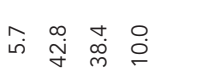 & & $\stackrel{\substack{+\dot{+}}}{\stackrel{\infty}{\infty}}$ & & 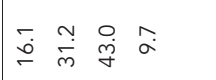 & & 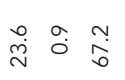 & & \begin{tabular}{ll} 
N \\
\multirow{y}{*}{}
\end{tabular} & & $\begin{array}{c}\infty \\
\infty \\
\infty\end{array} \stackrel{\circ}{\circ}$ \\
\hline & $=$ & & 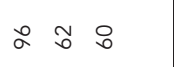 & & $m \propto \infty \infty$ & & $\approx \stackrel{\infty}{\check{\infty}}$ & & $\stackrel{m}{\infty} \stackrel{\infty}{\infty} \infty \stackrel{\infty}{\sim}$ & & 䓃 $\sim \stackrel{\text { 号 }}{\sim}$ & & $\infty \stackrel{\infty}{\wedge}$ & & ฉ \\
\hline$\cong$ న & $\circ$ & & 䒺㝘 & & 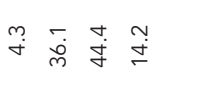 & & 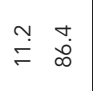 & & 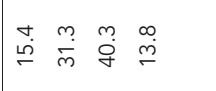 & & $\stackrel{\sigma}{\dot{m}} \stackrel{\circ}{-} \underset{\infty}{\stackrel{\infty}{\infty}}$ & & $\begin{array}{l}\stackrel{n}{n} \\
\dot{s} \\
\stackrel{m}{m}\end{array}$ & & 守 \\
\hline$\overline{0}$ & $=$ & & 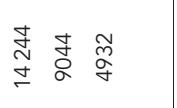 & & 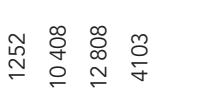 & & 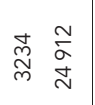 & & 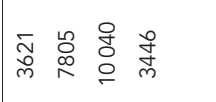 & & $\stackrel{n}{\bar{\sigma}} \bar{\alpha} \underset{\sim}{\stackrel{\infty}{\infty}} \underset{\sim}{\infty}$ & & 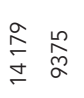 & & 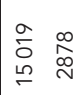 \\
\hline 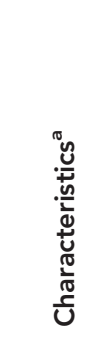 & & 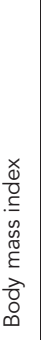 & 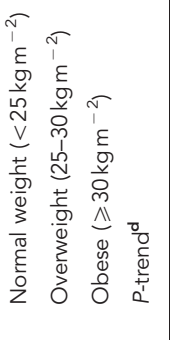 & 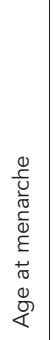 & 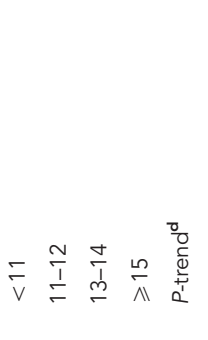 & 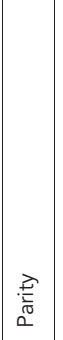 & 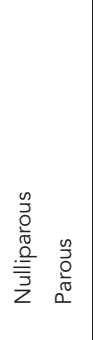 & 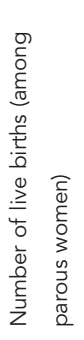 & 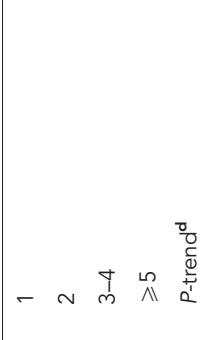 & 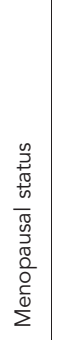 & 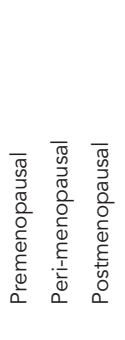 & 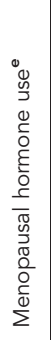 & 产 & 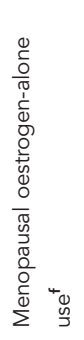 & 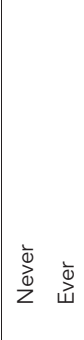 \\
\hline
\end{tabular}




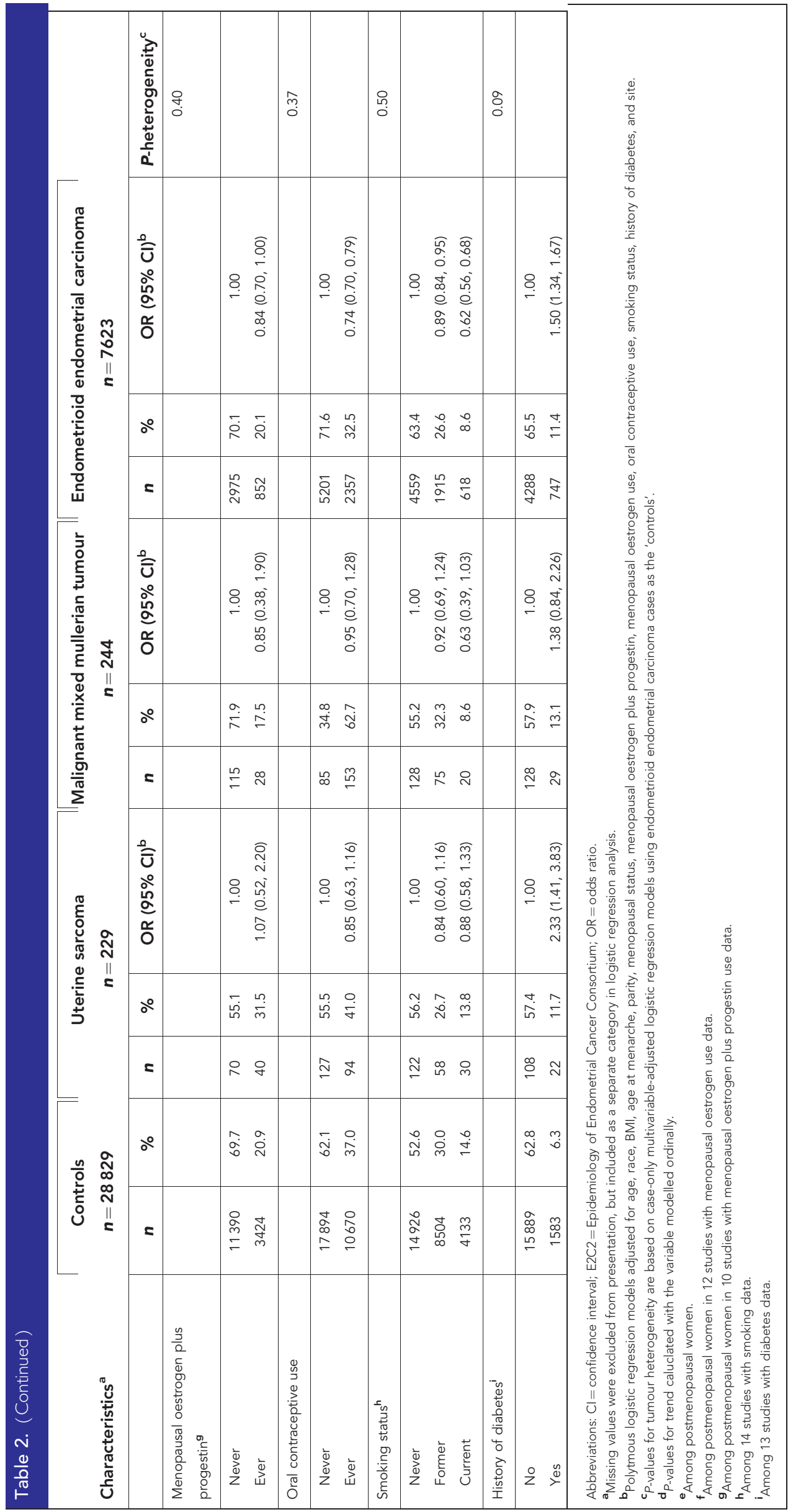


Table 3. Adjusted ORs and $95 \%$ Cls of risk factors for histological subtypes of uterine sarcoma, based on a pooled analysis of 15 observational studies in the $\mathrm{E} 2 \mathrm{C} 2$

Histological subtypes of uteirne sarcoma

Characteristics ${ }^{\mathrm{a}}$

Controls Endometrial stromal sarcoma $\quad$ Leiomyosarcoma

\begin{tabular}{|c|c|c|c|c|c|c|c|c|c|}
\hline & $n$ & $\%$ & $\mathbf{n}$ & $\%$ & OR $(95 \% \mathrm{Cl})^{\mathrm{b}}$ & $\mathbf{n}$ & $\%$ & OR $(95 \% \mathrm{Cl})^{\mathrm{b}}$ & $\boldsymbol{P}$-heterogeneity ${ }^{c}$ \\
\hline Body mass index & & & & & & & & & 0.39 \\
\hline Normal weight $\left(<25 \mathrm{~kg} \mathrm{~m}^{-2}\right)$ & 14244 & 49.4 & 42 & 42.9 & 1.00 & 33 & 40.2 & 1.00 & \\
\hline Overweight $\left(25-30 \mathrm{~kg} \mathrm{~m}^{-2}\right)$ & 9044 & 31.4 & 26 & 26.5 & $1.02(0.62,1.68)$ & 20 & 24.4 & $0.90(0.51,1.60)$ & \\
\hline Obese $\left(\geqslant 30 \mathrm{~kg} \mathrm{~m}^{-2}\right)$ & 4932 & 17.1 & 27 & 27.6 & $1.74(1.03,2.93)$ & 23 & 28.0 & $1.56(0.88,2.77)$ & \\
\hline$P$-trend ${ }^{d}$ & & & & & 0.07 & & & 0.26 & \\
\hline Age at menarche & & & & & & & & & 0.10 \\
\hline$<11$ & 1252 & 4.3 & 7 & 7.1 & 1.00 & 6 & 7.3 & 1.00 & \\
\hline $11-12$ & 10408 & 36.1 & 39 & 39.8 & $0.88(0.39,2.01)$ & 38 & 46.3 & $1.10(0.44,2.73)$ & \\
\hline $13-14$ & 12808 & 44.4 & 42 & 42.9 & $0.88(0.38,2.01)$ & 27 & 32.9 & $0.75(0.29,1.91)$ & \\
\hline$\geqslant 15$ & 4103 & 14.2 & 8 & 8.2 & $0.60(0.21,1.71)$ & 9 & 11.0 & $1.01(0.34,2.98)$ & \\
\hline$P$-trend $d^{d}$ & & & & & 0.41 & & & 0.44 & \\
\hline Parity & & & & & & & & & 0.40 \\
\hline Nulliparous & 3234 & 11.2 & 11 & 11.2 & 1.00 & 12 & 14.6 & 1.00 & \\
\hline Parous & 24912 & 86.4 & 81 & 82.6 & $0.97(0.51,1.83)$ & 65 & 79.3 & $0.76(0.40,1.44)$ & \\
\hline $\begin{array}{l}\text { Number of live births (among parous } \\
\text { women) }\end{array}$ & & & & & & & & & 0.17 \\
\hline 1 & 3621 & 14.5 & 8 & 9.9 & 1.00 & 13 & 20.0 & 1.00 & \\
\hline 2 & 7805 & 31.3 & 26 & 32.1 & $1.59(0.71,3.56)$ & 19 & 29.2 & $0.71(0.35,1.46)$ & \\
\hline $3-4$ & 10040 & 40.3 & 38 & 46.9 & $2.02(0.91,4.45)$ & 28 & 43.1 & $0.78(0.39,1.54)$ & \\
\hline$\geqslant 5$ & 3446 & 13.8 & 9 & 11.1 & $1.36(0.50,3.67)$ & 5 & 7.7 & $0.35(0.12,1.03)$ & \\
\hline$P$-trend ${ }^{d}$ & & & & & 0.31 & & & 0.12 & \\
\hline Menopausal status & & & & & & & & & 0.22 \\
\hline Premenopausal & 4015 & 13.9 & 23 & 23.5 & 1.00 & 25 & 30.5 & 1.00 & \\
\hline Peri-menopausal & 281 & 1.0 & 0 & 0.0 & NE & 2 & 2.4 & $0.73(0.12,4.41)$ & \\
\hline Postmenopausal & 23826 & 82.6 & 70 & 71.4 & $0.85(0.42,1.72)$ & 50 & 61.0 & $0.35(0.16,0.75)$ & \\
\hline Menopausal hormone use $e^{e}$ & & & & & & & & & 0.98 \\
\hline Never & 13412 & 58.5 & 26 & 40.6 & 1.00 & 27 & 54.0 & 1.00 & \\
\hline Ever & 9287 & 40.5 & 37 & 57.8 & $1.53(0.54,4.31)$ & 23 & 46.0 & $0.80(0.22,2.98)$ & \\
\hline Menopausal oestrogen-alone use $\mathrm{f}^{f}$ & & & & & & & & & 0.97 \\
\hline Never & 15019 & 76.9 & 40 & 63.5 & 1.00 & 32 & 78.0 & 1.00 & \\
\hline Ever & 2878 & 14.7 & 12 & 19.0 & $1.02(0.29,3.61)$ & 6 & 14.6 & $1.63(0.18,14.95)$ & \\
\hline $\begin{array}{l}\text { Menopausal oestrogen plus } \\
\text { progestin }\end{array}$ & & & & & & & & & 0.72 \\
\hline Never & 11390 & 69.7 & 26 & 47.3 & 1.00 & 21 & 55.3 & 1.00 & \\
\hline Ever & 3424 & 20.9 & 17 & 30.9 & $1.43(0.35,5.76)$ & 13 & 34.2 & $0.79(0.08,7.90)$ & \\
\hline Oral contraceptive use & & & & & & & & & 0.54 \\
\hline Never & 17894 & 62.1 & 52 & 53.1 & 1.00 & 44 & 53.7 & 1.00 & \\
\hline Ever & 10670 & 37.0 & 44 & 56.4 & $0.85(0.53,1.34)$ & 36 & 43.9 & $0.72(0.44,1.19)$ & \\
\hline Smoking status ${ }^{h}$ & & & & & & & & & 0.22 \\
\hline Never & 14926 & 52.6 & 50 & 56.2 & 1.00 & 41 & 50.6 & 1.00 & \\
\hline Former & 8504 & 30.0 & 21 & 23.6 & $0.66(0.39,1.11)$ & 28 & 34.6 & $1.15(0.70,1.90)$ & \\
\hline Current & 4133 & 14.6 & 16 & 18.0 & $1.09(0.61,1.94)$ & 9 & 11.1 & $0.75(0.36,1.56)$ & \\
\hline History of diabetes ${ }^{i}$ & & & & & & & & & 0.65 \\
\hline No & 15889 & 62.8 & 47 & 64.4 & 1.00 & 33 & 48.5 & 1.00 & \\
\hline Yes & 1583 & 6.3 & 11 & 15.1 & $2.28(1.02,5.12)$ & 10 & 14.7 & $1.91(0.77,4.77)$ & \\
\hline
\end{tabular}

Abbreviations: $\mathrm{Cl}=$ confidence interval; $\mathrm{E} 2 \mathrm{C2}=$ Epidemiology of Endometrial Cancer Consortium; $\mathrm{NE}=$ not estimable (due to zero cells); OR=odds ratio.

${ }^{a}$ Missing values were excluded from presentation, but included as a separate category in logistic regression analysis.

b Polytomous logistic regression models adjusted for age, race, BMl, age at menarche, menopausal status, menopausal oestrogen plus progestin, menopausal oestrogen use, oral contraceptive use, smoking status, history of diabetes, and site.

' $P$-values for tumour heterogeneity are based on case-only multivariable-adjusted logistic regression models using endometrial stromal sarcoma cases as the 'controls'.

$\mathbf{d}_{P \text {-values for trend caluclated with the variable modelled ordinally. }}$

e Among postmenopausal women.

f Among postmenopausal women in 12 studies with menopausal oestrogen use data.

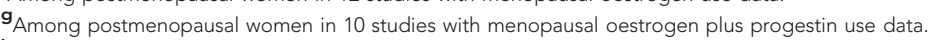

h Among 14 studies with smoking data.

'Among 13 studies with diabetes data. 
deregulation of the Wnt signalling pathway (Chiang and Oliva, 2011), while leiomyosarcomas are characterised by chromosome 1 deletion. The relationship between aetiologic risk factors and these molecular data is lacking, but this information would allow for a better understanding of uterine sarcoma tumour biology.

Our pooled analysis has several strengths, including the largest sample size of uterine sarcomas examined in the literature to date and availability of data on important risk factors and confounders. Several limitations of the current analysis should be noted. Although our sample size was large relative to previous studies, the histological subtype analyses were affected by small numbers as evidenced by large CIs. The ascertainment of exposure variables differed across studies, potentially introducing misclassification bias. Because of these differences, some variables were classified using crude categories to harmonise across studies. Importantly, we did not observe between-study statistical heterogeneity for any variable under consideration. We had insufficient data from the studies in the pooled analysis on other risk factors of interest, including infertility history, tamoxifen use, history of uterine fibroids, and previous cancer diagnoses. Other novel risk factors, including occupational exposures (Koivisto-Korander et al, 2012) and in vitro fertilisation (Venn et al, 2001), have been examined infrequently and should be studied in appropriate epidemiological settings. Disease misclassification is another possible bias given the potential for differential diagnosis of uterine cancer across diagnosis years, regions, and countries represented by the individual studies. Although MMMTs have recently been excluded from the uterine sarcoma classification, we expect a small proportion of these tumours to be misclassified as primary uterine sarcomas. Finally, this pooled analysis included cases and controls from diverse geographic regions, potentially introducing clinical heterogeneity in our study design. In conclusion, we provide evidence of common aetiologic pathways for EEC and uterine sarcoma. Further exploration of factors that might explain patterns of age- and race-specific incidence rates for uterine sarcoma are needed.

\section{ACKNOWLEDGEMENTS}

This research was supported by the Intramural Research Program of the National Cancer Institute, National Institutes of Health.

\section{REFERENCES}

Audet-Walsh E, Lepine J, Grégoire J, Plante M, Caron P, Têtu B, Ayotte P, Brisson J, Villeneuve L, Bélanger A, Guillemette C (2011) Profiling of endogenous estrogens, their precursors, and metabolites in endometrial cancer patients: association with risk and relationship to clinical characteristics. J Clin Endocrinol Metab 96(2): E330-E339.

Brinton LA, Sakoda LC, Sherman ME, Frederiksen K, Kjaer SK, Graubard BI, Olsen JH, Mellemkjaer L (2005) Relationship of benign gynecologic diseases to subsequent risk of ovarian and uterine tumors. Cancer Epidemiol Biomarkers Prev 14(12): 2929-2935.

Brooks SE, Zhan M, Cote T, Baquet CR (2004) Surveillance, epidemiology, and end results analysis of 2677 cases of uterine sarcoma 1989-1999. Gynecol Oncol 93(1): 204-208.

Calle EE, Kaaks R (2004) Overweight, obesity and cancer: epidemiological evidence and proposed mechanisms. Nat Rev Cancer 4(8): 579-591.

Chiang S, Oliva E (2011) Cytogenetic and molecular aberrations in endometrial stromal tumors. Hum Pathol 42(5): 609-617.

Chu PG, Arber DA, Weiss LM, Chang KL (2001) Utility of CD10 in distinguishing between endometrial stromal sarcoma and uterine smooth muscle tumors: an immunohistochemical comparison of 34 cases. Mod Pathol 14(5): 465-471.

D’Angelo E, Prat J (2010) Uterine sarcomas: a review. Gynecol Oncol 116(1): 131-139.
Dossus L, Rinaldi S, Becker S, Lukanova A, Tjonneland A, Olsen A, Stegger J, Overvad K, Chabbert-Buffet N, Jimenez-Corona A, Clavel-Chapelon F, Rohrmann S, Teucher B, Boeing H, Schütze M, Trichopoulou A, Benetou V, Lagiou P, Palli D, Berrino F, Panico S, Tumino R, Sacerdote C, Redondo ML, Travier N, Sanchez MJ, Altzibar JM, Chirlaque MD, Ardanaz E, Bueno-de-Mesquita HB, van Duijnhoven FJ, Onland-Moret NC, Peeters PH, Hallmans G, Lundin E, Khaw KT, Wareham N, Allen N, Key TJ, Slimani N, Hainaut P, Romaguera D, Norat T, Riboli E, Kaaks R (2010) Obesity, inflammatory markers, and endometrial cancer risk: a prospective case-control study. Endocr Relat Cancer 17(4): 1007-1019.

Harlow BL, Weiss NS, Lofton S (1986) The epidemiology of sarcomas of the uterus. J Natl Cancer Inst 76(3): 399-402.

Hosmer DaL. Stanley (2000) Applied Logistic Regression. John Wiley \& Sons, INC.: New York.

Jaakkola S, Lyytinen HK, Pukkala E, Ylikorkala O (2011) Use of estradiolprogestin therapy associates with increased risk for uterine sarcomas. Gynecol Oncol 122(2): 260-263.

Koivisto-Korander R, Martinsen JI, Weidepass E, Leminen A, Pukkala E (2012) Incidence of uterine leiomyosarcoma and endometrial stromal sarcoma in Nordic countries: results from NORDCAN and NOCCA databases. Maturitas 72(1): 56-60.

Kvale G, Heuch I, Ursin G (1988) Reproductive factors and risk of cancer of the uterine corpus: a prospective study. Cancer Res 48(21): 6217-6221.

Lavie O, Barnett-Griness O, Narod SA, Rennert G (2008) The risk of developing uterine sarcoma after tamoxifen use. Int J Gynecol Cancer 18(2): 352-356.

McCluggage WG (2002) Uterine carcinosarcomas (malignant mixed Mullerian tumors) are metaplastic carcinomas. Int J Gynecol Cancer 12(6): 687-690.

Oh JC, Wu W, Tortolero-Luna G, Broaddus R, Gershenson DM, Burke TW, Schmandt R, Lu KH (2004) Increased plasma levels of insulin-like growth factor 2 and insulin-like growth factor binding protein 3 are associated with endometrial cancer risk. Cancer Epidemiol Biomarkers Prev 13(5): 748-752.

Olson SH, Chen C, De Vivo I, Doherty JA, Hartmuller V, Horn-Ross PL, Lacey Jr JV, Lynch SM, Sansbury L, Setiawan VW, Schouten LJ, Shu XO (2009) Maximizing resources to study an uncommon cancer: E2C2-Epidemiology of Endometrial Cancer Consortium. Cancer Causes Control 20(4): 491-496.

Prat J (2009) FIGO staging for uterine sarcomas. Int J Gynaecol Obstet 104(3): 177-178.

Rosato V, Zucchetto A, Bosetti C, Dal Maso L, Montella M, Pelucchi C, Negri E, Franceschi S, La Vecchia C (2011) Metabolic syndrome and endometrial cancer risk.. Ann Oncol 22(4): 884-889.

Schwartz SM, Thomas DB (1989) A case-control study of risk factors for sarcomas of the uterus. The World Health Organization Collaborative Study of Neoplasia and Steroid Contraceptives. Cancer 64(12): 2487-2492.

Schwartz SM, Weiss NS (1990) Marital status and the incidence of sarcomas of the uterus. Cancer Res 50(6): 1886-1889.

Schwartz SM, Weiss NS, Daling JR, Gammon MD, Liff JM, Watt J, Lynch CF, Newcomb PA, Armstrong BK, Thompson WD (1996) Exogenous sex hormone use, correlates of endogenous hormone levels, and the incidence of histologic types of sarcoma of the uterus. Cancer 77(4): 717-724.

Schwartz SM, Weiss NS, Daling JR, Newcomb PA, Liff JM, Gammon MD, Thompson WD, Watt JD, Armstrong BK, Weyer P (1991) Incidence of histologic types of uterine sarcoma in relation to menstrual and reproductive history. Int J Cancer 49(3): 362-367.

Sherman ME, Devesa SS (2003) Analysis of racial differences in incidence, survival, and mortality for malignant tumors of the uterine corpus. Cancer 98(1): 176-186.

Skubitz KM, D’Adamo DR (2007) Sarcoma. Mayo Clin Proc 82(11): 1409-1432.

Venn A, Jones P, Quinn M, Healy D (2001) Characteristics of ovarian and uterine cancers in a cohort of in vitro fertilization patients. Gynecol Oncol 82(1): 64-68.

Wang T, Rohan TE, Gunter MJ, Xue X, Wactawski-Wende J, Rajpathak SN, Cushman M, Strickler HD, Kaplan RC, Wassertheil-Smoller S, Scherer PE, Ho GY (2011) A prospective study of inflammation markers and endometrial cancer risk in postmenopausal hormone nonusers. Cancer Epidemiol Biomarkers Prev 20(5): 971-977.

Weiderpass E, Persson I, Adami HO, Magnusson C, Lindgren A, Baron JA (2000) Body size in different periods of life, diabetes mellitus, hypertension, and risk of postmenopausal endometrial cancer (Sweden). Cancer Causes Control 11(2): 185-192.

This work is published under the standard license to publish agreement. After 12 months the work will become freely available and the license terms will switch to a Creative Commons AttributionNonCommercial-Share Alike 3.0 Unported License. 